\title{
Prognostic Factors for Treatment Outcomes of First Line Chemotherapy in Metastatic Breast Cancer
}

\author{
Hanan Gamal-Eldin Mostafa
}

Department of Clinical Oncology, Faculty of Medicine, Assiut University, Assiut, Egypt

\begin{abstract}
Background: A large number of old and new chemotherapeutic agents are now used in the treatment of metastatic breast cancer (MBC) patients. The choice of chemotherapy regimen depends on several factors concerning not only the tumor's characteristics, but also the patient preference, so the clinician must identify the prognostic factors that may help in tailoring treatment to each patient. The aim of the study was to evaluate the prognostic factors for response, time to tumor progression (TTP) and overall survival (OS) after the first line chemotherapy for MBC.

Patients and Methods: The study included 30 eligible women who had measurable metastatic disease and prior anthracycline based treatment in the adjuvant setting. All patients received cisplatin $75 \mathrm{mg} / \mathrm{m} 2 \mathrm{on}$ day 1 and gemcitabine $1000 \mathrm{mg} / \mathrm{m} 2$ on days $1 \& 8$ of 21 -day-cycles to a maximum 8 cycles. Prognostic factors studied were performance status (P.S), age, grade, menopausal status, hormonal receptor status, disease free interval (DFI) and number of metastatic sites.

RESULTS: Partial response was found in $43.3 \%$ (13) of the patients. Median TTP was 10 months. Median OS was 12 months. Prognostic factors significant for response were performance status $(\mathrm{P}=0.04)$, number of metastatic sites $(\mathrm{P}=0.02)$ and disease free interval $(\mathrm{P}=0.002)$. Prognostic factor for TTP was performance status $(\mathrm{P}=0.03)$, for overall survival performance status $(0.009)$ and hormonal receptor $(\mathrm{P}=0.008)$.

Conclusion: Prior to the initiation of first line chemotherapy for metastatic breast cancer, it is necessary to evaluate the performance status, number of metastatic sites and disease free interval to tailor treatment to achieve maximum benefit for the patients.
\end{abstract}

Key words: Metastatic breast cancer, gemcitabine plus cisplatin, prognostic factors.

Corresponding author: Hanan Gamal

E-mail: mostafahanan36@yahoo.com

\section{INTRODUCTION}

Breast cancer, the most frequent malignancy in women, is a global problem and a leading cause of cancer mortality ${ }^{1}$. Median survival from diagnosis of metastatic disease is 2 to 3 years, with $5 \%$ to $10 \%$ survival beyond 5 years $^{2}$.

The treatment goal in women with advanced breast cancer include prolongation of life, control of tumor burden, reduction in cancer-related symptoms or complications and maintenance of quality of life and function ${ }^{3}$.

Cytotoxic chemotherapy remains a mainstay of treatment for women with breast cancer, irrespective of hormonal-receptor status and is the backbone of many novel treatments incorporating biological therapy. Chemotherapy has substantial side effects including fatigue, nausea, vomiting, myelosuppresion, neuropathy, diarrhea and alopecia. For this reason, treatment of women with chemotherapy for advanced disease involves tradeoffs between palliation and toxicity of therapy ${ }^{4}$.
The widespread inclusion of anthracyclins in the adjuvant setting and concerns regarding cardiotoxicity limit their use as first-line therapy in metastatic disease. During the past decade, other cytotoxic drugs with activity in advanced breast cancer were identified, including taxanes, gemcitabine, vinorelbine and capecitabine $^{5}$.

Among several agents that have been used in metastatic breast cancer, gemcitabine and platinum compounds have been well-characterized single agents $^{6,7}$. Synergism between gemcitabine (a compound that inhibits DNA repair) and cisplatin (a compound that induces DNA damage) has been demonstrated in vitro studies. Exposure to gemcitabine can counteract the cisplatin resistance that results from the up-regulation of DNA repair processes. Cisplatin enhances the rate of incorporation of gemcitabine leading to apoptosis ${ }^{8}$.

Clinicaly, these agents have partially non-overlapping toxicity, as gemcitabine does not enhance cisplatin- 
induced nephrotoxicity or neurotoxicity and cisplatin causes only mild myelosuppresion ${ }^{9}$.

Well established clinical factors can predict the likelihood of response to therapy and long-term treatment outcomes in women with metastatic breast cancer. Patients who have received less therapy, have a longer disease-free interval since initial diagnosis, soft tissue or bone metastasis, fewer symptoms and better performance status are more likely to experience longer survival with metastatic disease than heavily treated patients with shorter treatment intervals, visceral metastasis and greater symptomatology ${ }^{3}$.

The most relevant prognostic factors for predicting survival from the time of metastatic occurrence are age at initial diagnosis, hormonal receptor status and site of metastasis. It is also shown that the metastases interval is an easily assessed and valuable multifactorial prognostic index reflecting the multiparametric variability of the disease $^{10}$.

A prognostic factor is defined as a measurement taken at the time of diagnosis or surgery that is associated with outcome (e.g. overall survival, disease-free survival or local control). They are used to estimate outcome following specific systemic therapy. Mathematically a prognostic factor is demonstrated as a statistically (and clinically) significant separation of curves outcome that are based on the presence or absence of the factors in a Cox proportional hazard ratio ${ }^{3}$.

The aim of the study was to identify the significant prognostic factors for treatment outcome of first line chemotherapy (gemcitabine and cisplatin) in metastatic breast cancer. The prognostic factors may assist physicians in evaluation of patients and in directing them toward the appropriate therapeutic decision.

\section{PATIENTS AND METHODS}

The study included all patients with metastatic breast cancer who presented to the Clinical Oncology department, Assiut University Hospital between March 2006 to September 2008 (30 eligible cases). All the patients were evaluable for response, toxicity and prognostic factors. The median follow-up time was 12 months (range 6-24 months).

\section{Eligibility Criteria:}

Eligible women had metastatic breast carcinoma with the following criteria: minimum of one bidimensionally measurable lesion by radiographs, Eastern Cooperative Oncology Group performance status ( $0-2)$, adequate bone marrow reserve and liver and renal functions. The cancer must be metastatic after adjuvant anthracycline-based regimen. Prior gemcitabine or cisplatin was not allowed, nor was any prior chemotherapy for metastatic disease.

\section{Study Design:}

All patients received cisplatin $75 \mathrm{mg} / \mathrm{m} 2$ intravenous infusion over 1 hour on day 1 with pre-hydration measures and antiemetics plus gemcitabine $1000 \mathrm{mg} /$ $\mathrm{m} 2$ intravenous infusion over 30 minutes on day 1 and 8 . Chemotherapy was given every 21 days for a maximum of 8 cycles or until disease progression, intolerable toxicity, or patient withdrawal.

\section{Study Evaluation:}

Before the study, patients underwent complete medical history, physical examination and evaluation of performance status.

Radiological evaluation included chest x-ray or computed tomography (CT), abdominal ultrasound or CT to identify visceral disease and bone scan. $\mathrm{CT}$ or magnetic resonance of the central nervous system was symptom driven. Radiographic evaluation was performed before initial protocol treatment and repeated after the initial 3 cycles and then after every two subsequent treatment cycles.

Complete blood counts before treatment and every treatment cycle were done.

Response was assessed according to WHO criteria. A complete response (CR), partial response (PR) or stable disease (SD) was confirmed 4 weeks after first observation of the response. Time to tumor progression was calculated from the initiation of treatment to the first observation of disease progression. Overall survival was calculated from the start of treatment to the date of death or last visit date.

Toxicity was evaluated by National Cancer Institute Toxicity Criteria version 2 after each cycle.

\section{Statistical Methods:}

This trial was designed to identify the significant prognostic factors for response, time to tumor progression (TTP) and overall survival (OS).

Time to tumor progression and overall survival were assessed using Kaplan-Meier method.11

Prognostic factors for OS, TTP and response rate (RR) were analyzed by use of Cox regression analyses.

\section{RESULTS}

Thirty women entered this study and all of them were assessable for efficacy and toxicity. All patients had been 
previously treated with anthracycline-based regimen as adjuvant treatment.

The median age of the patients was 49 years (range: 30-60 years). The median follow-up duration was 12 months (range: 6-24 months)

\section{Table (1) shows patient characteristics.}

The outcomes of patients treated with cisplatin and gemcitabine was as follow: PR was achieved in 13 patients out of $30(43.3 \%)$, ten patients $(33.3 \%)$ had SD and 7 patients $(23.3 \%)$ had PD.

The most common grade $3 / 4$ toxicities included thrombocytopenia in 6 patients $(20 \%)$, nausea and vomiting in 6 patients $(20 \%)$, neutropenia in 4 patients(13\%), anemia in one patient $(3 \%)$ and febrile neutropenia in one patient $(3 \%)$. There was no treatment related deaths.

For metastasis, the lung was the most common metastatic site (14 patients, $46.6 \%$ ), bone (13 patients, $43 \%$ ), skin and soft tissue (12 patients, 40\%), liver (7 patients, 23\%), lymph nodes (3 patients, 10\%) and brain (2 patients, $8 \%$ ).
Table (2) shows the univariate analysis of prognostic factors for response. Performance status $(p=0.04)$, number of metastatic site $(p=0.02)$ and disease free interval $(p=0.002)$ were significant prognostic factors for response.

Table (3) shows prognostic factors for time to progression. Performance status was the only prognostic factor for TTP $(p=0.03)$.

Table (4) shows the prognostic factors for overall survival. Performance status (P.S) $(p=0.009)$ and hormonal receptor positive $(\mathrm{P}=0.008)$ were significant prognostic factors for OS.

Figure (1) shows the median time to progression (10 months, 95\% CI 8.2-11.8) and one year time to tumor progression was $14 \%$.

Figure (2) shows the median overall survival (12 months, 95\% CI 11.2-12.8). One and two years overall survivals were $43.5 \%$ and $10.9 \%$, respectively. One and two years disease free survivals were $48.5 \%$ and $5 \%$, respectively.

Table 1: Patient characteristics.

\begin{tabular}{lcc}
\hline Characters & Frequency & \% \\
\hline Performance Status (Eastern Cooperative Oncology Group) & 15 & 50.0 \\
0 & 8 & 26.7 \\
1 & 7 & 23.3 \\
2 & & 53.3 \\
\hline Menopausal status & 16 & 46.7 \\
Pre & 14 & 56.7 \\
POST & & 43.3 \\
\hline Hormonal status & 17 & 46.7 \\
Negative & 13 & 53.3 \\
Positive & & \\
\hline Tumor grade & 14 & 60.0 \\
2 & 16 & 40.0 \\
3 & 18 & 3.3 \\
\hline Disease free interval & 12 & 36.7 \\
$<1$ year & 11 & 53.3 \\
$>1$ year & 11 & 6.7 \\
\hline Number of metastases & 16 & 3.3 \\
1 & 2 & \\
2 & 11 & \\
3 & & \\
4 & & \\
\hline
\end{tabular}


Kasr-El-Aini Journal Of Clinical Oncology And Nuclear Medicine

Vol. 7 | No. 1-2

Hanan Gamal-Eldin.

Table 2: Univariate analysis for prognostic factors for response to gemcitabine plus cisplatin as first line regimen for metastatic breast cancer.

\begin{tabular}{|c|c|c|c|c|c|}
\hline & & \multicolumn{3}{|c|}{ Response } & \multirow{2}{*}{ p-value } \\
\hline & & PD & PR & SD & \\
\hline \multirow{4}{*}{ Performance Status } & \multirow{2}{*}{$0+1$} & 4 & 12 & 7 & \multirow{4}{*}{$0.04 *$} \\
\hline & & $44.2 \%$ & $92.3 \%$ & $70.0 \%$ & \\
\hline & \multirow{2}{*}{2} & 3 & 1 & 3 & \\
\hline & & $42.9 \%$ & $7.7 \%$ & $30.0 \%$ & \\
\hline \multirow{4}{*}{ grade } & \multirow{2}{*}{2} & 3 & 6 & 5 & \multirow{4}{*}{$0.95 *$} \\
\hline & & $21.4 \%$ & $42.9 \%$ & $35.7 \%$ & \\
\hline & \multirow{2}{*}{3} & 4 & 7 & 5 & \\
\hline & & $25 \%$ & $43.8 \%$ & $31.2 \%$ & \\
\hline \multirow{6}{*}{ No of metastasis site } & \multirow{2}{*}{1} & 1 & 5 & 5 & \multirow{6}{*}{$0.02 *$} \\
\hline & & $9.1 \%$ & $45.5 \%$ & $45.5 \%$ & \\
\hline & \multirow{4}{*}{3} & 3 & 8 & 5 & \\
\hline & & $18.8 \%$ & $50 \%$ & $31.2 \%$ & \\
\hline & & 3 & 0 & 0 & \\
\hline & & $100 \%$ & 0 & 0 & \\
\hline \multirow{4}{*}{ Menopausal status } & \multirow{2}{*}{ Pre } & 5 & 5 & 6 & \multirow{4}{*}{$0.3^{*}$} \\
\hline & & $31.2 \%$ & $31.2 \%$ & 37.55 & \\
\hline & \multirow{2}{*}{ post } & 2 & 8 & 4 & \\
\hline & & 14.35 & $57.1 \%$ & $28.4 \%$ & \\
\hline Age & & $44.29 \pm 7.3$ & $48 \pm 10.4$ & $46.4 \pm 10.2$ & $0.4 * *$ \\
\hline Disease free interval & & $10 \pm 1.5$ & $24 \pm 1.2$ & $12 \pm 1.5$ & $0.002 * * *$ \\
\hline
\end{tabular}

*fisher exact test, $* *$ Kruskal-Wallis test, $* * * \log$ rank test

Table 3: Prognostic factors for time to progression (Kaplan Meir analysis) of 30 metastatic breast cancer patients treated by gemcitabine plus cisplatin as first line regimen.

\begin{tabular}{|c|c|c|c|c|c|c|}
\hline & & \multirow{2}{*}{ Median } & \multirow{2}{*}{ Std. Error } & \multicolumn{2}{|c|}{ 95\% Confidence Interval } & \multirow{2}{*}{$\begin{array}{c}\text { P-value } \\
\text { Log Rank } \\
\text { (Mantel-Cox }\end{array}$} \\
\hline & & & & Lower Bound & Upper Bound & \\
\hline \multirow{2}{*}{ grade } & 2 & 10 & 0.73 & 8.57 & 11.43 & \multirow{2}{*}{0.58} \\
\hline & 3 & 10 & 1.83 & 6.40 & 13.59 & \\
\hline \multirow{2}{*}{ Hormonal receptor } & negative & 8 & 0.91 & 6.20 & 9.79 & \multirow{2}{*}{0.07} \\
\hline & positive & 10 & 1.04 & 7.95 & 12.04 & \\
\hline \multirow{2}{*}{ Performance status } & 1 & 10 & 0.51 & 8.99 & 11.08 & \multirow{2}{*}{0.03} \\
\hline & 2 & 8 & 0.61 & 6.79 & 9.21 & \\
\hline \multirow{2}{*}{ Number of metastatic sites } & 1 & 11 & 2.02 & 7.02 & 14.97 & \multirow{2}{*}{0.3} \\
\hline & 2 & 10 & 0.67 & 8.68 & 11.31 & \\
\hline
\end{tabular}

Table 4: Prognostic factors for overall survival (Kaplan Meir analysis) of 30 metastatic breast cancer patients treated by gemcitabine plus cisplatin as first line regimen.

\begin{tabular}{|c|c|c|c|c|c|c|}
\hline & & \multirow{2}{*}{ Median } & \multirow{2}{*}{ Std. Error } & \multicolumn{2}{|c|}{ 95\% Confidence Interval } & \multirow{2}{*}{$\begin{array}{c}\text { P-value } \\
\text { Log Rank } \\
\text { (Mantel-Cox) }\end{array}$} \\
\hline & & & & Lower Bound & Upper Bound & \\
\hline \multirow{2}{*}{ grade } & 2 & 12 & 2.38 & 7.32 & 16.67 & \multirow{2}{*}{0.8} \\
\hline & 3 & 12 & 0.93 & 10.17 & 13.82 & \\
\hline \multirow{2}{*}{ Hormonal receptor } & negative & 12 & 0.60 & 10.80 & 13.19 & \multirow{2}{*}{0.008} \\
\hline & positive & 18 & 1.75 & 14.56 & 21.43 & \\
\hline \multirow{2}{*}{ Performance status } & 1 & 13 & 1.29 & 10.45 & 15.54 & \multirow{2}{*}{0.009} \\
\hline & 2 & 10 & 1.19 & 7.65 & 12.34 & \\
\hline \multirow{2}{*}{$\begin{array}{l}\text { Number of metastatic } \\
\text { sites }\end{array}$} & 1 & 12 & 0.82 & 10.38 & 13.61 & \multirow{2}{*}{0.3} \\
\hline & 2 & 12 & 1.32 & 9.41 & 14.58 & \\
\hline
\end{tabular}




Vol. 7 | No. 1-2 $2011 \quad$ Prognostic Factors for Treatment Outcomes

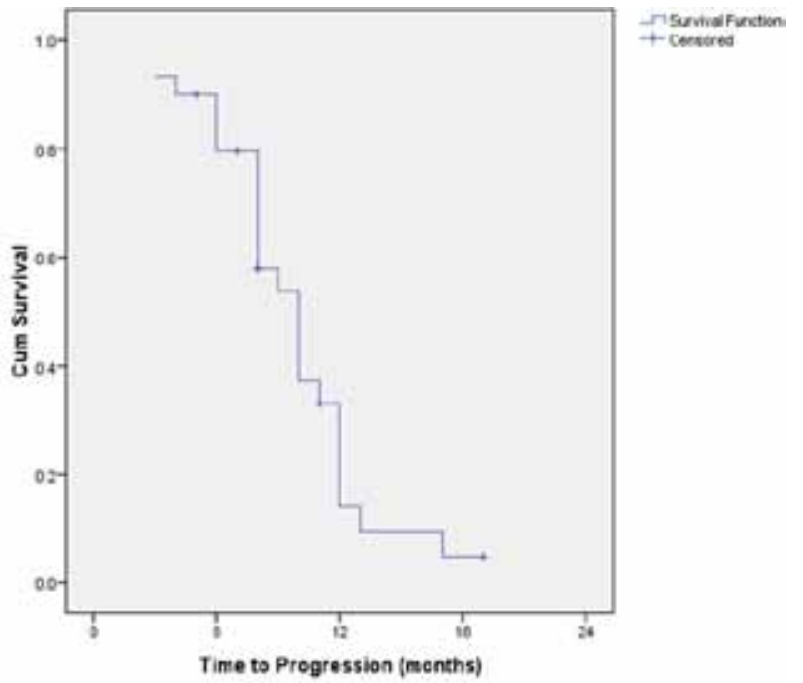

Figure 1: Time to progression (TTP) of 30 metastatic breast cancer patients treated by gemcitabine plus cisplatin as first line regimen, Median TTP 10 months 95\% CI (8.2-11.8), One year $\mathrm{TTP}=14.2 \%$

\section{DISCUSSION}

Metastatic breast cancer (MBC) is a highly heterogenous disease where particular criteria must be considered, taking into account not only the clinical and biological parameters but also patient expectation and preference. The objectives of an optimal chemotherapy $\mathrm{CT}$ for $\mathrm{MBC}$ are to prolong survival and enhance the quality of life with minimal toxicity. The therapeutic strategy may include sequential single agents or combination CT. ${ }^{12,13}$.

In daily practice, the choice of CT regimen depends on several factors concerning not only the tumor's characteristics but also the patient, so the clinician must consider the following: if the patient underwent prior CT (including previous adjuvant treatment); if she has a good performance status, or any co-morbidities, as well as the toxicity profiles: the organization of schedule and the disease's characteristics as relapse-free interval from adjuvant therapy, clinical aggressiveness, sites of metastasis. Finally, but no less important, the clinician also must consider the patient's preference ${ }^{14}$.

Cisplatin-gemcitabine is a synergistic chemotherapy combination highly proven in a broad spectrum of epithelial neoplasms and shows a non-cross-resistance profile with the most active drugs in metastatic breast cancer ${ }^{15}$. The US Food and Drug Administration approved an indication for gemcitabine therapy for women with advanced breast cancer based on the highly significant prolongation of time to progression (TTP) ${ }^{16}$ and interim promising overall survival (OS) ${ }^{17}$.

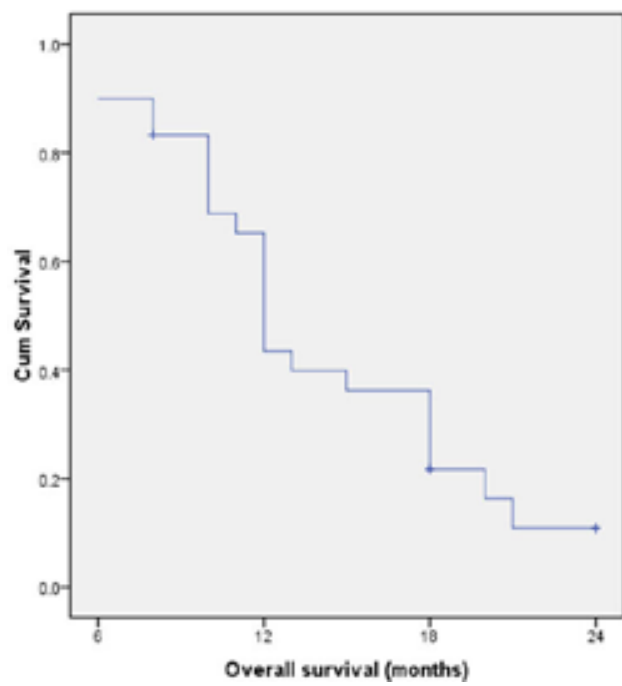

Minvisalfuncton

Figure 2: Overall survival (OS) of 30 metastatic breast cancer patients treated by gemcitabine plus cisplatin as first line regimen, Median OS 12 months 95\% CI (11.2-12.8), One year survival rate $=43.5 \%$, 2 year survival rate $=10.9 \%$.

The toxicity profile is an important factor in determining optimal combination therapy: balancing of efficacy and safety is a key goal for delivering a positive risk-benefit profile for each patient. Gemcitabine, capecitabine and docetaxel are highly active agents in anthracycline-pretreated metastatic breast cancer. A phase III trial compared gemcitabine plus docetaxel versus capecitabine plus docetaxel providing valuable information for a clinician in choosing an optimal treatment. This study showed that gemcitabine plus docetaxel is an active regimen in advanced breast cancer with similar efficacy to capecitabine plus docetaxel, but the toxicity profile favours gemcitabine plus docetax $l^{18}$.

Mostpatientswithadiagnosisofadvancedbreastcancer present having received previous adjuvant treatment. The adjuvant chemotherapy is particularly important for the choice of the optimal first chemotherapeutic approach to advanced disease ${ }^{14}$. The relationship between the activity of first line chemotherapy for $\mathrm{MBC}$ and prior adjuvant treatment is controversial, since some studies showed a poorer outcome ${ }^{19}$ whereas others demonstrated an outcome similar to patients who had not received previous adjuvant therapy ${ }^{20}$.

The site(s) and degree of metastatic dissemination are among the principle prognostic factors for patients with MBC. Patients with visceral metastasis to the liver and/or lung have a very poor prognosis. Although good performance status (P.S), restricted disease dissemination and limited extent of metastasis infiltration are associated with higher response to chemotherapy, responses are generally short lived, with rapid disease progression 
after treatment failure. Thus novel strategies for the management of patients with $\mathrm{MBC}$ with visceral metastasis are urgently needed ${ }^{21}$.

In this study, a response rate (RR) of $43.3 \%$, a median survival of 12 months and a median TTP of 10 months were achieved. Variable treatment outcomes of gemcitabine-cisplatin (GP) combination have been reported by others. A response rate of $54.5 \%$, a median OS of 14.8 months and a TTP of 8 months has been reported by Mohran ${ }^{22}$. Burch et al. ${ }^{23}$ reported RR of $29 \%$, OS 13 months and a TTP 7 months. Kim et al24 reported RR $28.9 \%$, median OS 19.5 months and a TTP 5.2 months. Wang et al. ${ }^{25}$ reported a RR of $62.2 \%$, a TTP 6.2 months. The difference in RR and OS observed among these studies, including this study, most likely reflect patient selection with good prognostic factors as longer disease free interval (DFI), higher percentage of patients who had one metastatic site, hormonal receptor positive and HER-2 negative. The lower RR in the study of Burch ${ }^{23}$ and Kim et al. ${ }^{24}$ are due to the use of GP combination as a second line treatment for metastatic disease.

The incidence of hematological toxicity in this trial was lower than that reported in other combination chemotherapy regimens such as gemcitabine plus taxol5 and capecitabine plus docetaxe ${ }^{26}$ combination which led to a $7 \%$ occurrence of grade 3 hand-foot syndrome. So the modest toxicity profile of GP combination regimen favors its use in the first line treatment of MBC.

For the prognostic factors, P.S, number of metastatic site and disease free interval (DFI) were prognostic factors for response. This is inagreement with Kramer et al. ${ }^{27}$ who reported that DFI $(\mathrm{p}=.009)$ and multiple sites of visceral metastasis $(p=.037)$ were significant prognostic factors for response in multivariate analysis.

In this study, P.S was the only significant prognostic factor for TTP; Kim et al. ${ }^{24}$ reported the same result.

Koshy et al28 reported a reduced risk of progression for triple negative (lack expression of estrogen, progesterone and HER-2 neu receptors) compared to non-triple negative MBC.

In this study, prognostic factor for survival were P.S and hormonal receptor status. This is inagreement with the results of Wheler et al. ${ }^{29}$, but Largiller et al. ${ }^{10}$ and Kramer et al. ${ }^{28}$ added other prognostic factors for OS as age at initial diagnosis, hormonal receptor status, multiple sites of visceral disease and disease free interval.

Liu et al. $^{30}$ reported that Karnofsky P.S, grade, estrogen receptor status, stage, number of lymph nodes, liver metastasis, DFI, number of metastasis had a significant impact on survival.

Bai et al. ${ }^{31}$ reported that P.S $>1$, multiple brain metastasis without whole brain irradiation in combination with chemotherapy were associated with poor prognosis.

Chew et al. ${ }^{32}$ analyzed polymorphism in genes in relation to gemcitabine-cisplatin combination treatment in metastatic breast cancer. They concluded that polymorphism was significantly associated with clinical outcomes and may tailor which patients benefit from this regimen.

\section{CONCLUSION}

Prior to the initiation of first line chemotherapy for patients with metastatic breast cancer and previous anthracycline based adjuvant therapy, it is necessary to evaluate the performance status, disease free interval and number of metastatic sites as prognostic factors for treatment outcomes. This study implicates the importance of searching for new prognostic and predictive factors and underscores the importance of the validated markers and patients characteristics in metastatic breast cancer that may assist the clinician to tailor treatment to achieve maximum benefit for the patients.

\section{REFERENCES}

1. Hortobagyi GN, de la Garza Salazar J, Pritchard K, Amadori D, Haidinger R, Hudis CA, et al. The global breast cancer burden: Variations in epidemiology and survival. Clin.Breast Cancer. 2005 Dec;6(5):391-401.

2. Ellis M, Hayes D, Lippman M. Treatment of metastatic breast cancer. In: Harris J, Lippman M, Morrow M, Osborne C, editors. Diseases of the breast. 2nd ed. Philadelphia: Lippincott Co.; 2000. p. 749-97.

3. Burstein HJ, Harris JR, Morrow M. Malignant tumors of the breast. In: DeVita VT, Lawrence TS, Rosenberg SA, editors. DeVita, Hellman and Rosenberg's cancer: Principles and practice of oncology. 8th ed. Philadelphia, Pa: Lippincott Williams \& Wilkins; 2008. p. 1606-54.

4. Mayer EL, Burstein HJ. Chemotherapy for metastatic breast cancer. Hematol.Oncol.Clin.North Am. 2007 Apr;21(2):257-72.

5. Albain KS, Nag SM, Calderillo Ruiz G, Jordaan JP, Llombart AC, Pluzanska A, et al. Gemcitabine plus paclitaxel versus paclitaxel monotherapy in patients with metastatic breast cancer and prior anthracycline treatment. J.Clin.Oncol. 2008;26(24):3950-7.

6. Seidman AD. Monotherapy options in the management of metastatic breast cancer. Semin.Oncol. 2003 Apr;30(2 Suppl 3):6-10.

7. Heinemann V. Role of gemcitabine in the treatment 
of advanced and metastatic breast cancer. Oncology 2003;64(3):191-206.

8. Achanta G, Pelicano H, Feng L, Plunkett W, Huang P. Interaction of $\mathrm{p} 53$ and DNA-PK in response to nucleoside analogues: Potential role as a sensor complex for DNA damage. Cancer Res. 2001 Dec 15;61(24):8723-9.

9. Nagourney RA, Link JS, Blitzer JB, Forsthoff C, Evans SS. Gemcitabine plus cisplatin repeating doublet therapy in previously treated, relapsed breast cancer patients. J.Clin.Oncol. 2000;18(11):2245-9.

10. Largillier R, Ferrero JM, Doyen J, Barriere J, Namer M, Mari V, et al. Prognostic factors in 1038 women with metastatic breast cancer. Ann.Oncol. 2008;19(12):2012-9.

11. Kaplan EL, Meier P. Non-parametric estimation from incomplete observations. J.Am.Stat.Assoc. 1985;53:22932 .

12. Briest S, Stearns V. Chemotherapeutic strategies for advanced breast cancer. Oncology 2007;21(11):1325-35.

13. Chang AE, Skibber JM. Evidence-based management of breast cancer. Oncology. An evidence-based approach. USA: Springer; 2006. p. 951-82.

14. Pronzato P, Rondini M. First line chemotherapy of metastatic breast cancer. Ann.Oncol. 2006;17(Suppl. 5):v165-8.

15. Sánchez Escribano Morcuende R, Alés Martínez JE, Aramburo González PM. Low dose Gemcitabine plus cisplatin in a weekly-based regimen as salvage therapy for relapsed breast cancer after taxane-anthracyclinecontaining regimens. Clin.Transl.Oncol. 2007;9(7):45964.

16. O'Shaughnessy J, Nag S, Calderillo Ruiz G, et al. Gemcitabine plus paclitaxel versus paclitaxel as first-line treatment for anthracycline-pretreated metastatic breast cancer: Interim results of a global phase III study. Proc. Am.Soc.Clin.Oncol. 2003;22(7a):Abstr. 25.

17. Albain KS, Nag S, Calderillo Ruiz G, et al. Global phase III study of gemcitabine plus paclitaxel (GT) vs. paclitaxel (T) as frontline therapy for Metastatic Breast Cancer (MBC): First report of overall survival. J Clin Oncol 2004;22(Suppl. 14S):510.

18. Chan S, Romieu G, Huober J, Delozier T, Tubiana Hulin M, Lluch A, et al. Gemcitabine plus Docetaxel (GD) versus Capecitabine plus Docetaxel (CD) for anythracycline pretreated Metastatic Breast Cancer (MBC) patients(pts): Results of a European Phase III study. J.Clin.Oncol. 2005;23(16S):581.

19. Pierga JY, Asselain B, Jouve M, Dieras V, Carton M, Laurence V, et al. Effect of adjuvant chemotherapy on outcome in patients with metastatic breast carcinoma treated with first-line doxorubicin-containing chemotherapy. Cancer 2001 Mar 15;91(6):1079-89.

20. Kardinal CG, Perry MC, Korzun AH, Rice MA, Ginsberg S, Wood WC. Responses to chemotherapy or chemohormonal therapy in advanced breast cancer patients treated previously with adjuvant chemotherapy. A subset analysis of CALGB Study 8081. Cancer $1988 \mathrm{Feb}$ $1 ; 61(3): 415-9$.

21. Yardley DA. Visceral disease in patients with metastatic breast cancer: Efficacy and safety of treatment with ixabepilone and other chemotherapeutic agents. Clin. Breast Cancer 2010;10(1):64-73.

22. Mohran TZ. Gemcitabine and cisplatin combination chemotherapy as a first-line treatment in patients with metastatic breast cancer. J.Egypt.Natl.Cancer Inst. 2004 Mar;16(1):8-14.

23. Burch PA, Mailliard JA, Hillman DW, Perez EA, Krook JE, Rowland KM, et al. Phase II study of gemcitabine plus cisplatin in patients with metastatic breast cancer: A North Central Cancer Treatment Group Trial. Am.J.Clin.Oncol. 2005 Apr;28(2):195-200.

24. Kim JH, Oh SY, Kwon HC, Lee S, Kim SH, Kim DC, et al. Phase II study of gemcitabine plus cisplatin in patients with anthracycline- and taxane-pretreated metastatic breast cancer. Cancer Res. Treat. 2008 Sep;40(3):101-5.

25. Wang Z, Hu X, Chen L, Wang J, Wang H, Wang L, et al. Efficacy of gemcitabine and cisplatin (GP) as firstline combination therapy in patients with triple-negative metastatic breast cancer: Preliminary results report of a phase II trial. J.Clin.Oncol. 2010;28(15s):Abstr. 1100.

26. Michalaki V, Gennatas S, Gennatas K. Low-dose capecitabine plus docetaxel as first-line therapy for metastatic breast cancer: Phase Il results. Anti-Cancer Drugs 2009;20(3):204-7.

27. Kramer JA, Curran D, Piccart M, de Haes JC, Bruning P, Klijn J, et al. Identification and interpretation of clinical and quality of life prognostic factors for survival and response to treatment in first-line chemotherapy in advanced breast cancer. Eur.J.Cancer 2000 Aug;36(12):1498-506.

28. Koshy N, Quispe D, Shi R, Mansour R, Burton GV. Cisplatin-gemcitabine therapy in metastatic breast cancer: Improved outcome in triple negative breast cancer patients compared to non-triple negative patients. Breast 2010;19(3):246-8.

29. Wheler J, Tsimberidou AM, Moulder S, Cristofanilli M, Hong D, Naing A, et al. Clinical outcomes of patients with breast cancer in a phase I clinic: The M. D. Anderson cancer center experience. Clin.Breast Cancer 2010;10(1):46-51.

30. Liu MT, Huang WT, Wang AY, Huang CC, Huang CY, Chang TH, et al. Prediction of outcome of patients with metastatic breast cancer: Evaluation with prognostic factors and Nottingham prognostic index. Support.Care Cancer 2010;18(12):1553-64.

31. Bai B, Yuan ZY, Liu DG, Teng XY, Wang SS. Clinical features and survival analysis of different subtypes of patients with breast cancer brain metastases. Chin.J.Cancer 2010;29(4):413-9.

32. Chew HK, Doroshow JH, Frankel P, Margolin KA, Somlo G, Lenz HJ, et al. Phase II studies of gemcitabine and cisplatin in heavily and minimally pretreated metastatic breast cancer. J.Clin.Oncol. 2009;27(13):2163-9. 